\title{
Management of patients with multiple myeloma in the era of COVID-19 pandemic: a consensus paper from the European Myeloma Network (EMN)
}

\author{
Evangelos Terpos $\mathbb{B}^{1} \cdot$ Monika Engelhardt ${ }^{2} \cdot$ Gordon Cook $^{3} \cdot$ Francesca Gay ${ }^{4} \cdot$ Maria-Victoria Mateos $\mathbb{D}^{5,6}$. \\ loannis Ntanasis-Stathopoulos $\mathbb{D}^{1} \cdot$ Niels W. C. J. van de Donk ${ }^{7} \cdot$ Hervé Avet-Loiseau $^{8} \cdot$ Roman Hajek $^{9}$. \\ Annette Juul Vangsted $\mathbb{D}^{10} \cdot$ Heinz Ludwig $\mathbb{D}^{11} \cdot$ Sonja Zweegman ${ }^{7} \cdot$ Philippe Moreau $^{12} \cdot$ Hermann Einsele $^{13} \cdot$ \\ Mario Boccadoro ${ }^{4}$ - Jesus San Miguel $^{14} \cdot$ Meletios A. Dimopoulos $^{1} \cdot$ Pieter Sonneveld $^{15}$
}

Received: 29 April 2020 / Revised: 6 May 2020 / Accepted: 13 May 2020 / Published online: 22 May 2020

(c) The Author(s), under exclusive licence to Springer Nature Limited 2020

\begin{abstract}
Patients with multiple myeloma (MM) seem to be at increased risk for more severe COVID-19 infection and associated complications due to their immunocompromised state, the older age and comorbidities. The European Myeloma Network has provided an expert consensus statement in order to guide therapeutic decisions in the era of the COVID-19 pandemic. Patient education for personal hygiene and social distancing measures, along with treatment individualization, telemedicine and continuous surveillance for early diagnosis of COVID-19 are essential. In countries or local communities where COVID-19 infection is widely spread, MM patients should have a PCR test of nasopharyngeal swab for SARS-CoV-2 before hospital admission, starting a new treatment line, cell apheresis or ASCT in order to avoid ward or community spread and infections. Oral agent-based regimens should be considered, especially for the elderly and frail patients with standard risk disease, whereas de-intensified regimens for dexamethasone, bortezomib, carfilzomib and daratumumab should be used based on patient risk and response. Treatment initiation should not be postponed for patients with end organ damage, myeloma emergencies and aggressive relapses. Autologous (and especially allogeneic) transplantation should be delayed and extended induction should be administered, especially in standard risk patients and those with adequate MM response to induction. Watchful waiting should be considered for standard risk relapsed patients with low tumor burden, and slow biochemical relapses. The conduction of clinical trials should continue with appropriate adaptations to the current circumstances. Patients with MM and symptomatic COVID-19 disease should interrupt anti-myeloma treatment until recovery. For patients with positive PCR test for SARS-CoV-2, but with no symptoms for COVID-19, a 14-day quarantine should be considered if myeloma-related events allow the delay of treatment. The need for surveillance for drug interactions due to polypharmacy is highlighted. The participation in international COVID-19 cancer registries is greatly encouraged.
\end{abstract}

\section{Introduction}

The coronavirus disease 2019 (COVID-19) has been rapidly evolved into a pandemic around the globe, since its emergence as an outbreak in Wuhan, China in late 2019. The

These authors contributed equally: Evangelos Terpos, Monika Engelhardt

Evangelos Terpos

eterpos@med.uoa.gr

Extended author information available on the last page of the article clinical presentation of the infection with the novel coronavirus SARS-CoV-2 varies significantly among the individuals and ranges from completely asymptomatic cases to potentially lethal respiratory infection and multi-organ damage [1]. The lack of herd immunity renders the population vulnerable to SARS-CoV-2 infection, whereas the case-fatality rate is not unanimous among different countries [2]. Increased age (65 years and above, especially 75 years and above), male gender, obesity and the presence of comorbidities are strong predictors of adverse outcomes among hospitalized patients with COVID-19 [3].

Accumulating data have consistently shown that patients with cancer are at increased risk for more severe COVID-19 infection and associated complications. In a hospital-based 
cohort of 338 patients with COVID-19 including 105 with coexistent malignancies, those with cancer had poorer survival $(p=0.02)$, had a higher chance of having at least one critical or severe manifestation $(p<0.01)$ and were more frequently admitted at the intensive care unit $(p=0.01)$ compared with those without any malignancy [4]. In another retrospective study including 28 cancer patients with COVID-19, the administration of antineoplastic treatment within 14 days from COVID-19 diagnosis was significantly associated with the risk for severe disease (HR = $4.08, p=0.037$ ) [5]. Chemotherapy or cancer-related surgery within 30 days from COVID-19 diagnosis has also been associated with increased risk for severe disease ( $\mathrm{OR}=5.34, p=0.0026)$ [6]. Despite various methodological limitations mainly due to the retrospective design, a meta-analysis of available studies has confirmed these observations [7]. For this reason, PCR screening for COVID-19 has been proposed in patients with lung cancer who are going to start a new treatment and this may be used more extensively in other cancers soon [8].

\section{Vulnerability of patients with multiple myeloma}

Multiple myeloma (MM) is a plasma cell dyscrasia characterized by the malignant proliferation of clonal plasma cells. The overproduction of a monoclonal component consisting of dysfunctional immunoglobulin, along with the suppressed levels of normal immunoglobulin classes, and the dysfunctional cellular and innate immunity, is the basis for the suboptimal immunological response of the host against pathogens. Renal impairment that is a frequent complication of the disease further contributes to the dysfunction of immune system and the development of infections and sepsis in MM [9]. Moreover, the current drug armamentarium against MM encompasses agents with substantial hematological toxicity, including neutropenia and lymphopenia [10]. Infections are a major source for morbidity and mortality among patients with MM [11-13]. Clinical studies have shown that patients under treatment with immunomodulatory drugs (IMiDs) and proteasome inhibitors (PIs) are at increased risk of severe infections [14, 15]. PIs impair T-cell function and are associated with varicella zoster virus (VZV) reactivation. Although CD38 directed monoclonal antibodies reduce immunosuppressive regulatory $\mathrm{T}$ cells, they also deplete natural killer (NK) cells and increase the susceptibility to viral and bacterial infections [16, 17]. High dose of steroids and high-dose melphalan with autologous stem cell transplantation (ASCT) further contribute to a immunocompromised state. Therefore, vaccination and prophylaxis with antivirals and antibiotics has been integrated in the management of patients with MM [18, 19].
Furthermore, patients with MM are typically older adults with several age-related comorbidities that have a significant impact on disease outcomes [20]. The multifactorial burden of cardiovascular disease is also substantial and may be aggravated by preexisting conditions, disease complications and drug toxicities [21]. In addition, individuals with $\mathrm{MM}$ are at an increased risk for venous thromboembolism (VTE) that may vary according to patient-, diseaseor treatment-related characteristics [22].

Although there is limited published evidence regarding the characteristics and outcomes of patients with $\mathrm{MM}$ and COVID-19 infection [23], clinical experience is growing rapidly in the field. Older age, male gender, obesity and comorbidities, mainly including immune impairment, cardiovascular disease, lung impairment and diabetes, have been repeatedly reported as risk factors among patients with COVID-19 [1, 3, 24, 25]. SARS-CoV-2 infection promotes a hypercoagulation state, as well [26]. Taking all the above into consideration, it is clear that patients with MM present with an increased risk of COVID-19 related complications with a potentially fatal outcome.

In this context, international and national recommendations have been formulated in order to guide physicians in the management of patients with MM. A summary of currently available recommendations is presented in Table 1 [27-33]. Although the general principles seem to coincide, there are aspects that have to be elucidated. Therefore, the European Myeloma Network (EMN) deemed it useful to provide updated guidance on the management of patients with MM in the era of COVID-19 pandemic (Fig. 1), while recognizing the need for generating prospective evidencebased myeloma-specific data.

\section{General recommendations}

In the era of the COVID-19 pandemic, we should care for our patients by minimizing their risk for infection without lowering our standards for providing them with the optimal therapeutic approach [34]. However, adaptations in our organization and prioritization of our clinical strategies are necessary in order to effectively confront the challenges in cancer care that are created by the pandemic [35-37]. Individualization of our strategy is essential and, apart from the well-established patient- and myeloma-related factors, we should also consider the current dynamics of the COVID-19 infection in the community.

Patient and caregiver education for disease prevention is of outmost importance. Hand hygiene and social distance are vital for preventing COVID-19 transmission, since there is no vaccine and no SARS-CoV-2-specific treatment available yet. Patients, caregivers and health professionals should be encouraged to wear a face mask when visiting the 


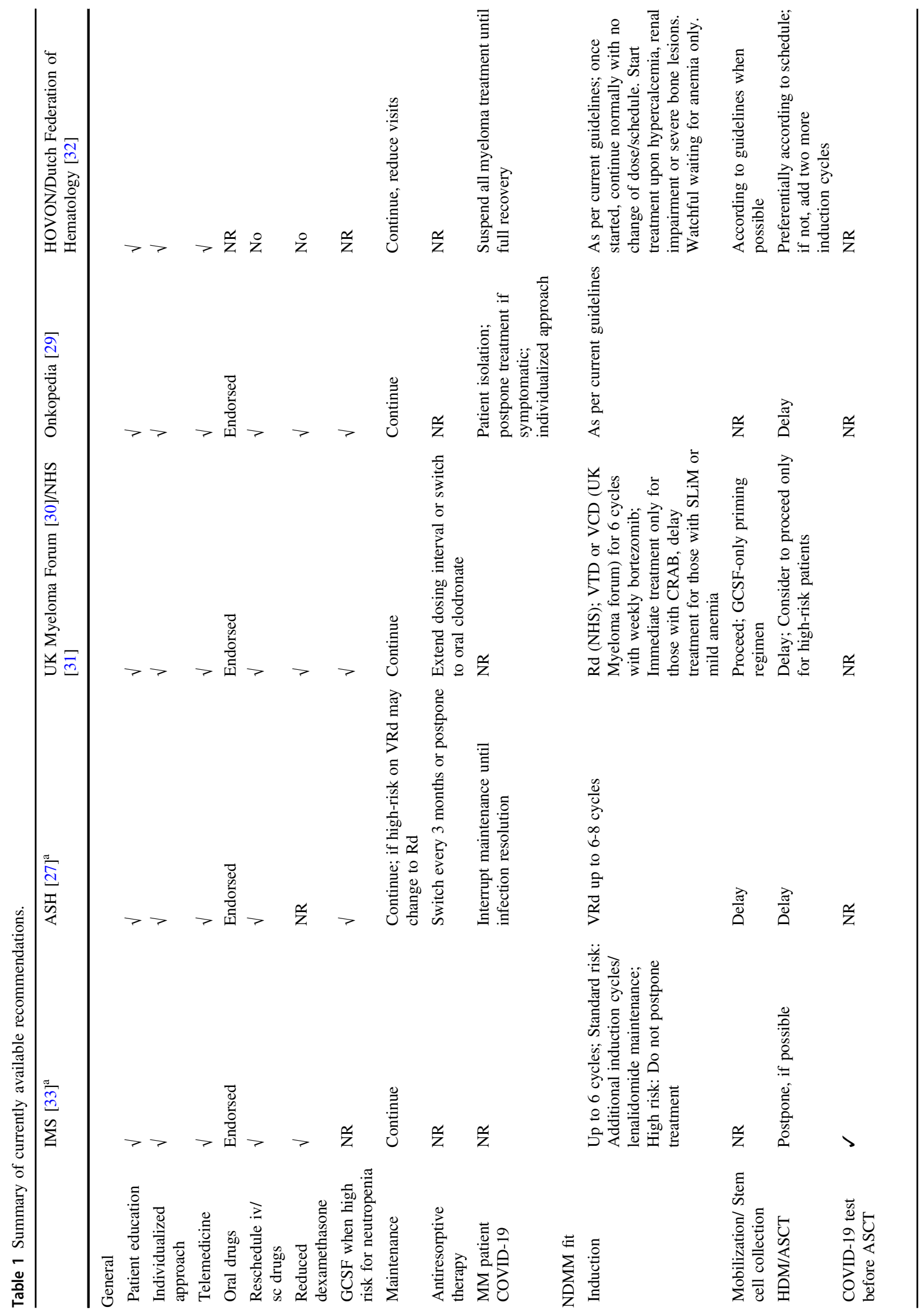




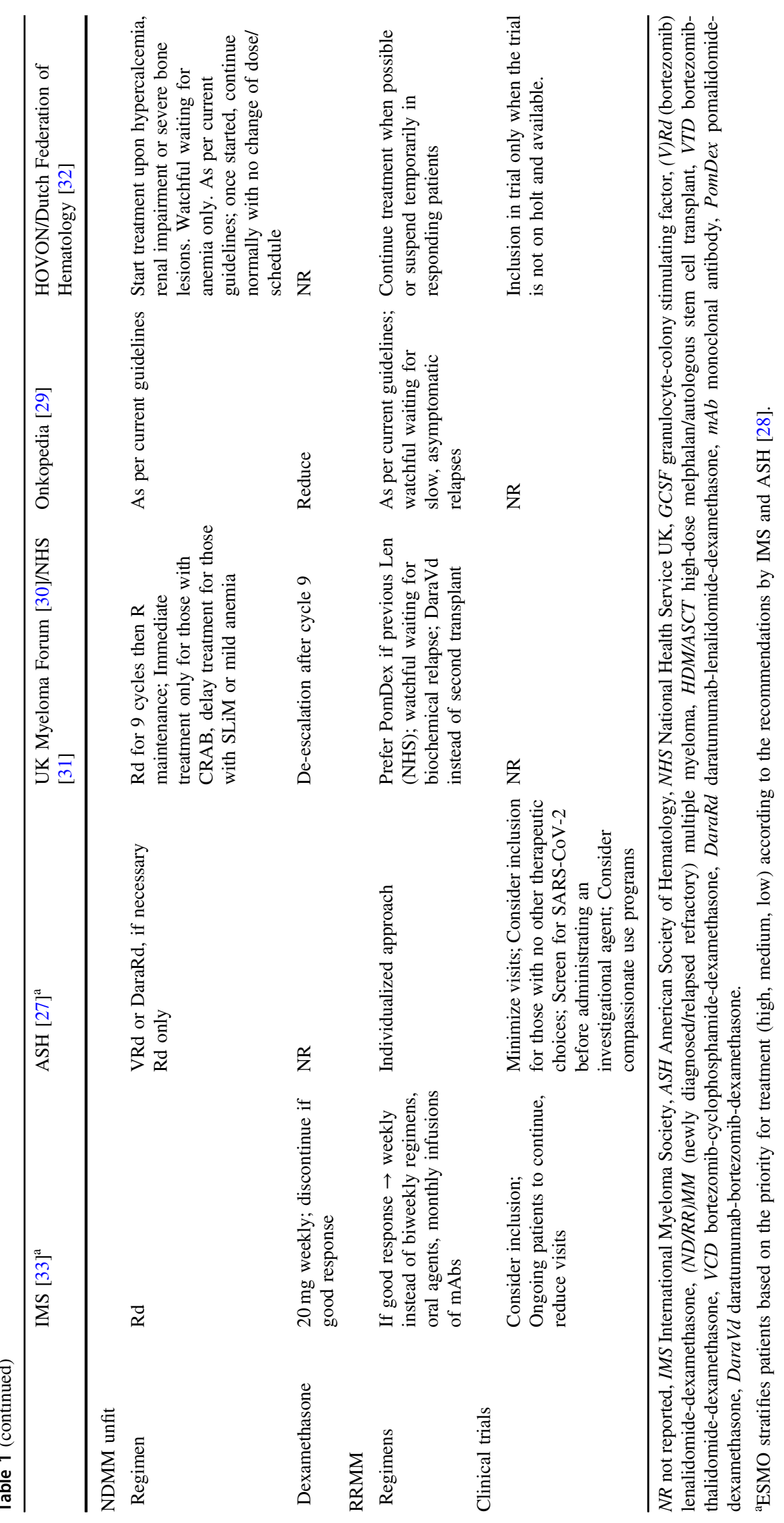




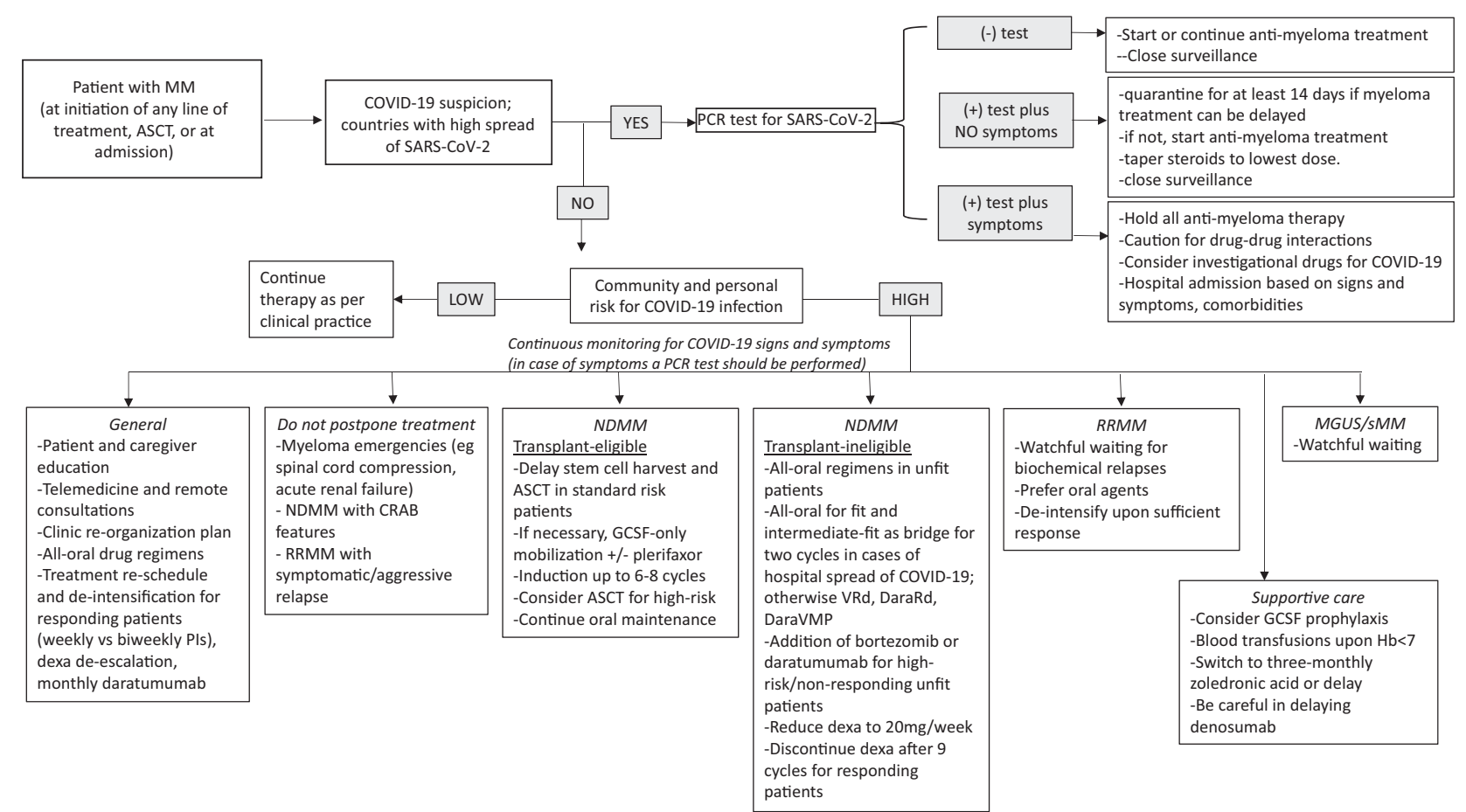

Fig. 1 Decision-making algorithm for the management of patients with MM in the era of the COVID-19 pandemic. In case of COVID-19 suspicion and a positive PCR test for SARS-CoV-2, treatment decisions should be made based on patient symptoms. A tailored approach is suggested based on the community and individual risk for COVID-19 infection.

clinic or going outside home. Respiratory masks are the best way to prevent the disease from spreading through the air via coughs or sneezes. Surgical masks are recommended to those who are more likely to contract or already have the infection to avoid spreading it further. Masks such as FFP2 and FFP3 are ideal for those who are not yet ill, or health care professionals taking precaution to prevent infection with SARS-CoV-2 when working or being near those who may have symptomatic disease. Even 'self-made cloth masks' have been proposed to be used for the entire community in several countries, when distancing cannot be achieved, especially in not open-air areas.

Telemedicine and novel technologies for remote communication are endorsed in order to reduce patient visits to the clinic. Blood examinations can also be preferred to be performed in a local laboratory instead of a high-volume hospital. In this case, caution should be made during the disease evaluation, because different laboratories may apply different techniques with distinct reference ranges, especially when determining free light chain levels. Unanticipated results should be confirmed in the reference laboratory.

Whenever possible, all-oral drug combinations should be considered over intravenous or subcutaneous agents in order to reduce visits to the clinic. However, this decision should be balanced with efficacy, providing the oral regimen is not inferior to the alternative intravenous scheme.
Treatment re-schedule and de-intensification can be considered for responding patients [38]. This may pertain to switching from twice to once weekly regimens (e.g., bortezomib and carfilzomib), monthly daratumumab infusions by omitting the bi-monthly phase, and dexamethasone deescalation. In the absence of previous infusion-related reactions, daratumumab may also be safely administered in 90 min under close monitoring [39]. A new subcutaneous formulation of daratumumab will reduce the injection time to $5 \mathrm{~min}$ and the rate of infusion reactions.

Treatment cannot be postponed in some cases of myeloma emergencies. Severe anemia and renal failure may necessitate hospitalization and immediate initiation of antimyeloma treatment along with supportive care [22]. Spinal cord compression may necessitate immediate initiation of radiotherapy and/or orthopedic decompensation [40, 41]. Orthopedic treatment of impeding fractures and radiotherapy for palliation of pain unresponsive to analgesics should not be postponed [40].

Continuous monitoring for COVID-19 signs and symptoms is highly recommended in order to prevent in-hospital dissemination of the disease. All patients should be screened for potential COVID-19-related signs and symptoms or contact with any person with suspected or confirmed COVID-19 infection, in order to be tested for SARSCoV-2. In countries or local communities where COVID-19 infection is widely spread, MM patients should have a PCR 
test of nasopharyngeal swab for SARS-CoV-2 before hospital admission, starting a new treatment line, cell apheresis or ASCT in order to avoid ward spread and infections; this strategy has been performed with striking success at centers with larger test capabilities [8]. For patients admitted into the ward (i.e., for ASCT) we should limited the rotation of the companion person, and ideally this person should have a negative SARS-CoV-2 PCR.

In cases of myeloma patients with positive PCR test for SARS-CoV-2, but with no symptoms for COVID-19 infection, a 14-day quarantine should be considered if myeloma-related events allow the delay of treatment (i.e., if the patient does not have acute renal impairment or extended bone disease with fractures, severe anemia or other features of aggressive disease). Otherwise, treatment should be given with very close monitor of the patients for the development of COVID-19 signs and symptoms. If the patient has symptoms of COVID-19 infection, antimyeloma treatment should be delayed until total recovery from COVID-19.

Testing of doctors and nurses is another important issue. Local guidelines should be followed; however, testing of health professionals not only in case of symptoms or contact with a known COVID-19 case is highly recommended, i.e., on a monthly basis until a vaccine against SARS-CoV-2 is ready for use.

\section{Patients with newly diagnosed multiple myeloma}

Treatment should not be delayed for newly diagnosed MM (NDMM) patients with active disease [42]. In particular, these patients have significant immune dysfunction but an uncontrolled malignant clone that has affected the host immunity long before the MM diagnosis is made in most cases. The overall tumor burden, as defined by laboratory and imaging studies, and the previous history of asymptomatic long-standing paraproteinemia should be also taken into consideration. Patients who are in the maintenance phase of their treatment should continue with their oral therapy and reduce visits to the clinic, whereas subcutaneous bortezomib administration might be omitted or delayed depending on the achieved depth of response and the risk of relapse.

\section{Transplant-eligible NDMM patients}

In view of the novel triplet (or quadruplet) upfront combinations for NDMM patients the necessity of upfront ASCT has been challenged [43]. In this context and due to the anticipated immunosuppression following ASCT, it is recommended to postpone mobilization, stem cell harvest, conditioning and ASCT, mainly in patients with standard risk disease. Physicians may completely avoid ASCT in patients with marginal fitness due to age or comorbidities. Stem cell harvest without ASCT should be considered for patients receiving daratumumab and or lenalidomide-based induction in order to achieve a sufficient stem cell yield [44, 45]. In this case, GCSF-only mobilization with the potential addition of plerifaxor should be considered in order to avoid the immunosuppressive effect of high-dose cyclophosphamide. However, in case of close contact with a person diagnosed with COVID-19, stem cell harvests and any transplant procedures should not be performed within at least 14 , and preferably 21 , days from the last contact.

Induction treatment can be administered for an extended period for up to 6-8 cycles. The combination of bortezomib with lenalidomide or thalidomide and dexamethasone (VRd or VTD), as well as the combination of daratumumab with VTD (DaraVTD) consist the preferred induction therapy [46-48]. The treatment schedule can be modified, for patients with sufficient response. Patients with high-risk disease features may receive ASCT after 6-8 induction cycles due to otherwise increased probability of progression. In this situation and in cases where the ASCT procedure cannot be postponed according to physician's discretion, exclusion of COVID-19 infection by PCR for SARS-CoV-2 is deemed necessary, along with strict precautions to prevent COVID-19 transmission in the transplantation department.

\section{Transplant ineligible NDMM patients}

Treatment for patients who are not eligible for transplant should be based on all-oral regimens, e.g., lenalidomide with dexamethasone (Rd), especially for unfit patients, whereas the addition of bortezomib or daratumumab can be considered for patients with high-risk disease, or for those without sufficient response to Rd. For fit or intermediate-fit myeloma patients $\mathrm{Rd}$ can be considered as a bridge for $2-3$ cycles, in case the COVID-19 pandemia is at a peak in the hospital; otherwise, the approved VRd or daratumumabbased therapies (DaraRd or DaraVMP) should be considered. Dexamethasone should be reduced to $20 \mathrm{mg}$ weekly, whereas de-escalation (or even interruption) should be considered for responding patients, especially after the completion of 9 cycles of treatment.

\section{Patients with relapsed/refractory MM}

Depending on the COVID-19 circumstances in the community, watchful waiting may be considered for biochemical relapses, especially for patients with a slow and gradual increase in the paraprotein level. However, 
patients with refractory disease, new onset of CRAB features or those with a biochemical relapse and a history of aggressive relapse with rapid deterioration of the clinical presentation should receive next-line treatment without delay.

Regarding the selection of treatment regimen, orally administered agents (ixazomib, lenalidomide, pomalidomide, and panobinostat) should be considered, if appropriate, again due to the patients' lesser need to be seen and treated at hospital/out-patient facilities. Neutropenia due to lenalidomide or pomalidomide has to be managed according to published recommendations [49]. Alternative therapeutic approaches are recommended instead of a salvage ASCT or an allogeneic transplant. Should a patient with relapsed/refractory MM (RRMM) achieve sufficient response [e.g., very good partial response (VGPR) or better], modifications in the treatment schedule are advisable (once weekly instead of twice weekly bortezomib/carfilzomib and monthly daratumumab infusions). Substitution of bortezomib or carfilzomib with ixazomib, in cases of VGPR or better, is not recommended, as it is not supported by clinical studies. There are no data for isatuximab once monthly and thus in cases of combination with pomalidomide and dexamethasone, in countries where the combination has been approved, the schedule of isatuximab administration has to remain unchanged (i.e., every two weeks) [50]. Similarly, elotuzumab in combination with pomalidomide and dexamethasone should be given according to protocol [51]. Selinexor or belantamab mafodotin can be used in triple-class refractory patients, if available $[52,53]$.

The need for continuous surveillance for any COVID-19related signs and symptoms among patients with RRMM is highlighted.

\section{Patients with monoclonal gammopathy of undetermined significance or smoldering MM}

Patients with a diagnosis of monoclonal gammopathy of undetermined significance or smoldering MM are typically in long-term follow-up and monitoring of their disease status. Although lenalidomide has provided encouraging results in patients at high-risk for progression [54, 55], this has not led to a change in the clinical practice outside clinical trials in Europe. Scheduled visits of patients with stable disease can be delayed with safety. Alternatively, blood examination in local laboratories and consultation via telemedicine is encouraged. It should be stressed that patients with high-risk disease should be carefully monitored for development of symptomatic disease requiring treatment [56].

\section{Supportive care}

Patients with a history of neutropenias and/or recurrent infections should receive prophylactic GCSF injections. Vaccination against influenza and pneumonococci species is highly recommended for all patients. Co-trimoxazole prophylaxis for Pneumonocystis jirovecii for all patients and levofloxacin prophylaxis for the first three months of treatment for NDMM patients are also highly recommended $[19,22]$. Acyclovir should be prescribed according to local protocols. Immunoglobulin administration may be given in an individualized basis, depending on the depth of suppression of polyclonal immunoglobulins and patient history of recurrent infections [20].

Patients with anemia who do not respond to treatment or are deteriorating should promptly receive erythropoiesis stimulating agents in order to prevent the need for blood transfusions and the visits to the clinic. The availability of blood supplies has been extensively restricted due to the reduction in blood donations during the COVID-19 pandemic [26].

SARS-CoV-2 has thombogenic potential and several patients with COVID-19 have developed thrombotic complications, including pulmonary embolism and even disseminated intravascular coagulation [26]. Antithrombosis prophylaxis in myeloma patients should be administered according to local or international guidelines [57, 58]. For countries with high incidence of COVID-19, lowmolecular-weight heparin (LMWH) may be considered over aspirin as thromboprophylaxis in myeloma patients under IMiD administration, irrespective of their thrombotic risk. Regarding the use of LMWH in myeloma patients with asymptomatic COVID-19 or with mild symptoms not requiring hospitalization, even in the absence of IMiD administration (i.e., while the MM patient is on quarantine and IMiD has been disrupted), several ongoing trials will reveal the prophylaxis value of LMWH in this setting.

Regarding antiresorptive treatment, patients on monthly zoledronic acid can switch to once every 3 months, especially for those with at least 1 year of prior therapy and those at first-line treatment who have achieved VGPR or better (and clinically stable bone status) [59, 60]. Denosumab doses may be administered at home if there is nursing facility at home in the specific country or the patient can be educated for self-administration. Long-term discontinuation of denosumab may result in a rebound effect and thus should be avoided [60].

\section{Clinical trials}

Although several clinical trials were initially halted or temporary suspended enrollment as a reaction to the global 
pandemic of COVID-19, gradual restoration of clinical trial activities are ongoing. Until then, determining the optimal bridging therapies in an individualized basis is essential. Studies with chimeric antigen receptor $\mathrm{T}$ cells or other immunotherapy strategies (i.e., bispecific T-cell engager, bispecific antibodies and cord blood CAR NK-cells) are usually paused during COVID-19 period, but they should be considered if they are the only option for the patients. Compassionate use and early access programs may be valuable alternatives.

Patients currently participating in clinical trials should continue treatment. Protocol modifications are deemed necessary in the context of reducing the amount and the duration of clinic visits, omitting visits exclusively for correlative studies, practicing telemedicine for safety assessments and disease evaluations, and shipping oral drugs to patients [61]. Several clinical trials have provided the option to switch from intravenous to subcutaneous daratumumab and reduce the infusion time of daratumumab to a minimum $[39,62]$. Patients with no other therapeutic choices receiving novel investigational agents in earlyphase clinical trials should be screened for COVID-19 infection before treatment administration. Close cooperation among the investigators, the sponsor and the local regulatory authorities is essential for all the above.

\section{Myeloma patients with COVID-19 infection}

Patients with MM and COVID-19 should be treated as per standard guidelines starting from isolation measures. Asymptomatic patients for COVID-19 should stay quarantined at home for at least 14 days, under close surveillance for detecting COVID-19-associated signs and symptoms, in cases where anti-myeloma therapy could be delayed. In patients with acute renal failure, extended bone disease, heavy anemia, or other aggressive myeloma features, treatment should be administered. If anti-myeloma treatment has been started, this might continue for patients with an asymptomatic COVID-19 infection and active myeloma (MM-related symptoms, new diagnosis, recent relapse, suboptimal response to treatment, e.g., less than VGPR), albeit pausing of anti-MM treatment should also be considered as an option. In this case, steroids and drugs inducing lymphopenia can be de-intensified.

Upon the emergence of symptomatic infection, treatment should be interrupted and steroids should be tapered to zero until full recovery from COVID-19, as is the case with influenza and other infections. Although symptomatic patients with mild COVID-19 disease may stay at home, close surveillance for aggravating symptoms is necessary. Upon such clinical suspicion, patient referral to a reference center for COVID-19 should not be delayed, because the clinical presentation may deteriorate rapidly and early intervention may be life-saving. For patients enrolled in a clinical trial, investigational agents should be interrupted until COVID-19 resolution and the reporting should abide with the corresponding guidelines. Usually, COVID-19 infection is reported as an adverse event of special interest, unless it fulfills the well-established criteria for a serious adverse event.

Patients with MM and COVID-19 infection can receive novel and investigational agents against SARS-CoV-2. A case report has shown that the use of a monoclonal antibody against interleukin-6, tocilizumab, provided significant clinical benefit to a patient with severe COVID-19 and underlying MM, possibly reducing the cytokine storm responsible for several symptoms of severe COVID-19 [23]. Clinical evidence of SARS-CoV-2 agent use for severe active infection in MM patients has also been made with remdesivir and other anti-viral drugs, tocilizumab, anakinra or convalescent plasma. Several trials with anticancer agents that are used in the treatment of patients with plasma cell neoplasms, i.e., selinexor, acalabrutinib, ibrutinib, are also recruiting patients with COVID-19.

Caution should be made due to polypharmacy. Patients with MM receive several drugs for supportive care along with drugs with direct anti-myeloma activity, whereas possible comorbidities may add to the list of concomitant medications. Many agents that are being evaluated against SARS-CoV-2, including antivirals, (hydroxy)chloroquine, azithromycin and remdesivir, may have significant interactions with other drugs and may result in significant hepatic, cardiac or renal toxicity $[18,63,64]$. Therefore, close monitoring of organ function is important.

\section{Concluding remarks}

COVID-19 pandemic has changed the way we live, behave and think, and has profound and multifaceted consequences in society, economy, politics, psychology, and health care systems. Optimizing our therapeutic approach in the era of this global infection is extremely challenging, as we have to balance the risk of a potentially lethal infection with the risk of a potentially lethal and incurable disease, taking into consideration the restricted resources, which are largely dedicated to COVID-19 services. Tailoring our treatment to each individual separately along with continuous surveillance for early diagnosis of COVID-19 infections among patients with $\mathrm{MM}$ are considered essential. Accumulating data in order to provide evidence-based guidance is of equal importance. In this context, we strongly encourage the participation in international registries collecting data on patients with MM or cancer in general and COVID-19 infection. EMN has such a project led by Gordon Cook, 
which looks at the spread of COVID-19 in myeloma patients and their outcome. The American Society of Hematology's Research Collaborative data hub COVID-19 Registry, the International Myeloma Society registry, and the international COVID-19 and Cancer Consortium database are other registries where myeloma patients can participate.

At this point, we stress that we adopted our guidelines according to the local situation and current knowledge. There are clearly countries (i.e., Germany, Greece, Czech Republic and others), where there are clearly less patients with COVID-19 infections and thus treatment is less intensively changed when compared with the international guidelines. In Germany for example, there is very low impact on clinical trial recruitment and performance, as stem cell collection and stem cell transplantation is ongoing without disruption. However, we will probably have COVID-19 for the next 1-2 years before the availability of any vaccination/therapy, but we will be able to handle it increasingly better. Thus, recommendations must be updated and revisited frequently, when data will be more mature regarding registry analyses and with more knowledge regarding the impact of various anti-myeloma therapy on COVID-19.

\section{Compliance with ethical standards}

Conflict of interest ET declares honoraria from BMS, Janssen, Celgene, Takeda, Genesis Pharma, Amgen and Novartis; research funding from Janssen, Amgen, Takeda and Genesis Pharma; ME declares no competing financial interests for this paper; GC declares Honoraria: Amgen, Bristol-Myers Squibb, Celgene, Janssen, Sanofi, Karyopharm and GSK; Research funding: Celgene, Janssen, Takeda; FG declares honoraria from BMS, Janssen, Celgene, Takeda, Amgen; advisory board from Janssen, Amgen, Takeda, Adaptive, Celgene, Oncopeptides, Roche, Abbvie, GSK; M-VM has received honoraria from lectures and boards from: Janssen, Celgene, Amgen, Takeda, Abbvie, GSK, Adaptive, Roche, Seattle Genetics; IN-S declares no competing financial interests for this paper; NWCJD has received research support from Janssen Pharmaceuticals, AMGEN, Celgene, Novartis, and BMS, serves in advisory boards for Janssen Pharmaceuticals, AMGEN, Celgene, BMS, Takeda, Roche, Novartis, Bayer, and Servier; HA-L declares no competing financial interests for this paper; $\mathrm{RH}$ has received research funding from Janssen, Amgen, Celgene, BMS, Novartis and Takeda, serves in advisory boards for Janssen, Amgen, Celgene, AbbVie, BMS, Novartis, PharmaMar and Takeda, and has received honoraria from Janssen, Amgen, Celgene, BMS, Pharma Mar and Takeda; AJV declares no competing financial interests for this paper; HL has received research funding from Takeda, Amgen, serves in Speaker's Bureau/Advisory Boards for Takeda, Amgen, Janssen, Celgene, Sanofi, Seattle Genetics and Bristol-Meyers. SZ has received research funding from Takeda and Janssen and serves in advisory boards for Celgene, Takeda, Janssen, Sanofi and Oncopeptides; PM declares honorarium form Celgene, Janssen, Takeda, Amgen and Abbvie; HE has received honoraria and declares consultancy for Janssen, Celgene, BMS, Amgen, Novartis and Takeda, and received research support from Janssen, Celgene, BMS, Amgen. MB has received honoraria from Sanofi, Celgene, Amgen, Janssen, Novartis, Bristol-Myers Squibb, and AbbVie; has received research funding from Sanofi, Celgene, Amgen, Janssen, Novartis, Bristol-Myers Squibb, and Mundipharma; JSM declares consultancy for Amgen, Bristol-Myers Squibb, Celgene, Janssen, MSD, Novartis, GSK Takeda, Sanofi, and Roche; MAD declares consultancy and honoraria from Janssen, Celgene, Takeda, Amgen and BMS; PS declares research funding from Amgen, Celgene, Janssen, Karyopharm, SkylineDx and Takeda and honoraria from Amgen, BMS, Celgene, Janssen, Karyopharm and Takeda.

Publisher's note Springer Nature remains neutral with regard to jurisdictional claims in published maps and institutional affiliations.

\section{References}

1. Wu Z, McGoogan JM. Characteristics of and important lessons from the coronavirus disease 2019 (COVID-19) outbreak in China: summary of a Report of 72314 Cases From the Chinese Center for Disease Control and Prevention. JAMA. 2020. https:// doi.org/10.1001/jama.2020.2648. [Epub ahead of print].

2. Dowd JB, Andriano L, Brazel DM, Rotondi V, Block P, Ding X, et al. Demographic science aids in understanding the spread and fatality rates of COVID-19. Proc Natl Acad Sci USA. 2020;117:9696-8.

3. Chen R, Liang W, Jiang M, Guan W, Zhan C, Wang T, et al. Risk factors of fatal outcome in hospitalized subjects with coronavirus disease 2019 from a nationwide analysis in China. Chest. 2020. pii: S0012-3692(20)30710-8. https://doi.org/10.1016/j.chest.2020. 04.010. [Epub ahead of print].

4. Dai M, Liu D, Liu M, Zhou F, Li G, Chen Z, et al. Patients with cancer appear more vulnerable to SARS-COV-2: a multi-center study during the COVID-19 outbreak. Cancer Discov. 2020. https://doi.org/10.1158/2159-8290.CD-20-0422. [Epub ahead of print].

5. Zhang L, Zhu F, Xie L, Wang C, Wang J, Chen R, et al. Clinical characteristics of COVID-19-infected cancer patients: a retrospective case study in three hospitals within Wuhan, China. Ann Oncol. 2020. pii: S0923-7534(20)36383-3. https://doi.org/10. 1016/j.annonc.2020.03.296. [Epub ahead of print].

6. Liang W, Guan W, Chen R, Wang W, Li J, Xu K, et al. Cancer patients in SARS-CoV-2 infection: a nationwide analysis in China. Lancet Oncol. 2020;21:335-7.

7. Desai A, Sachdeva S, Parekh T, Desai R. COVID-19 and cancer: lessons from a pooled meta-analysis. JCO Glob Oncol. 2020;6:557-9.

8. Passaro A, Peters S, Mok TSK, Attili I, Mitsudomi T, de Marinis F. Testing for COVID-19 in lung cancer patients. Ann Oncol. 2020. pii: S0923-7534(20)39293-0. https://doi.org/10.1016/j. annonc.2020.04.002. [Epub ahead of print].

9. Sorrig R, Klausen TW, Salomo M, Vangsted A, Gimsing P. Risk factors for infections in newly diagnosed Multiple Myeloma patients: A Danish retrospective nationwide cohort study. Eur J Haematol. 2019;102:182-90.

10. Teh BW, Harrison SJ, Pellegrini M, Thursky KA, Worth LJ, Slavin MA. Changing treatment paradigms for patients with plasma cell myeloma: impact upon immune determinants of infection. Blood Rev. 2014;28:75-86.

11. Blimark C, Holmberg E, Mellqvist UH, Landgren O, Bjorkholm M, Hultcrantz M, et al. Multiple myeloma and infections: a population-based study on 9253 multiple myeloma patients. Haematologica. 2015;100:107-13.

12. Holmstrom MO, Gimsing P, Abildgaard N, Andersen NF, Helleberg C, Clausen NA, et al. Causes of early death in multiple myeloma patients who are ineligible for high-dose therapy with 
hematopoietic stem cell support: a study based on the nationwide Danish Myeloma Database. Am J Hematol. 2015;90:E73-74.

13. Bringhen S, Offidani M, Palmieri S, Pisani F, Rizzi R, Spada S, et al. Early mortality in myeloma patients treated with firstgeneration novel agents thalidomide, lenalidomide, bortezomib at diagnosis: a pooled analysis. Crit Rev Oncol Hematol. 2018;130:27-35.

14. Chen M, Zhao Y, Xu C, Wang X, Zhang X, Mao B. Immunomodulatory drugs and the risk of serious infection in multiple myeloma: systematic review and meta-analysis of randomized and observational studies. Ann Hematol. 2018;97:925-44.

15. Basler M, Lauer C, Beck U, Groettrup M. The proteasome inhibitor bortezomib enhances the susceptibility to viral infection. J Immunol. 2009;183:6145-50.

16. Feng X, Zhang L, Acharya C, An G, Wen K, Qiu L, et al. Targeting CD38 suppresses induction and function of $\mathrm{T}$ regulatory cells to mitigate immunosuppression in multiple myeloma. Clin Cancer Res. 2017;23:4290-4300.

17. Nahi H, Chrobok M, Gran C, Lund J, Gruber A, Gahrton G, et al. Infectious complications and $\mathrm{NK}$ cell depletion following daratumumab treatment of Multiple Myeloma. PLoS ONE. 2019;14: $\mathrm{e} 0211927$.

18. Girmenia C, Cavo M, Offidani M, Scaglione F, Corso A, Di Raimondo F, et al. Management of infectious complications in multiple myeloma patients: expert panel consensus-based recommendations. Blood Rev. 2019;34:84-94.

19. Drayson MT, Bowcock S, Planche T, Iqbal G, Pratt G, Yong K, et al. Levofloxacin prophylaxis in patients with newly diagnosed myeloma (TEAMM): a multicentre, double-blind, placebo-controlled, randomised, phase 3 trial. Lancet Oncol. 2019;20:1760-72.

20. Engelhardt M, Ihorst G, Duque-Afonso J, Wedding U, SpatSchwalbe E, Goede V, et al. Structured assessment of frailty in multiple myeloma as a paradigm of individualized treatment algorithms in cancer patients at advanced age. Haematologica. 2020;105:1183-1188.

21. Bringhen S, Milan A, Ferri C, Wasch R, Gay F, Larocca A, et al. Cardiovascular adverse events in modern myeloma therapyincidence and risks. A review from the European Myeloma Network (EMN) and Italian Society of Arterial Hypertension (SIIA). Haematologica. 2018;1031422-32.

22. Terpos E, Kleber M, Engelhardt M, Zweegman S, Gay F, Kastritis E, et al. European Myeloma Network guidelines for the management of multiple myeloma-related complications. Haematologica. 2015;100:1254-66.

23. Zhang X, Song K, Tong F, Fei M, Guo H, Lu Z, et al. First case of COVID-19 in a patient with multiple myeloma successfully treated with tocilizumab. Blood Adv. 2020;4:1307-10.

24. Grasselli G, Zangrillo A, Zanella A, Antonelli M, Cabrini L, Castelli A, et al. Baseline characteristics and outcomes of 1591 Patients Infected With SARS-CoV-2 admitted to ICUs of the Lombardy Region, Italy. JAMA. 2020. https://doi.org/10.1001/ja ma.2020.5394. [Epub ahead of print], https://www.ncbi.nlm.nih. gov/pubmed/?term $=32250385$.

25. Goyal P, Choi JJ, Pinheiro LC, Schenck EJ, Chen R, Jabri A, et al. Clinical characteristics of Covid-19 in New York City. N Engl J Med. 2020. https://doi.org/10.1056/NEJMc2010419. [Epub ahead of print], https://www.ncbi.nlm.nih.gov/pubmed/?term $=32302078$.

26. Terpos E, Ntanasis-Stathopoulos I, Elalamy I, Kastritis E, Sergentanis TN, Politou M, et al. Hematological findings and complications of COVID-19. Am J Hematol. 2020. https://doi.org/10. 1002/ajh.25829. [Epub ahead of print].

27. Rajkumar S, Cavo M, Mikhail J, Mateos M, Jackson G, Moreau P, et al. COVID-19 and Multiple myeloma: frequently asked questions (Version 1.0; last reviewed April 7, 2020). https://www. hematology.org/covid-19/covid-19-and-multiple-myeloma Accessed 19 April 2020.
28. European Society of Medical Oncology. ESMO management and treatment adapted recommendations in the COVID-19 era: multiple myeloma. https://www.esmo.org/guidelines/cancer-patientmanagement-during-the-covid-19-pandemic/haematological-ma lignancies-multiple-myeloma-in-the-covid-19-era Accessed 19 April 2020.

29. von Lilienfeld-Toal M, Greinix H, Hirsch H, Na I-K, Sandherr M, Schanz U, et al. Coronavirus-Infektion (COVID-19) bei Patienten mit Blut- und Krebserkrankungen. Onkopedia. Version vom 15. https://www.onkopedia.com/de/onkopedia/guidelines/corona virus-infektion-covid-19-bei-patienten-mit-blut-und-krebserkra nkungen/@@guideline/html/index.html?chapter=6.2. 48\#ID0EASBG Accessed 19 April 2020.

30. UK Myeloma Forum guidance to support medical decisionmaking in the management of myeloma patients during the COVID-19 (Coronavirus) outbreak (25 March 2020). Accessed 19 April 2020.

31. Chemotherapy Clinical Reference Group UK. Interim treatment change options during the COVID-19 pandemic, endorsed by NHS England (20 April 2020). Accessed 20 April 2020.

32. HOVON/Dutch Federation of Hematology. Recommendations for patients with multiple myeloma during the COVID-19 epidemic [dutch]. http://www.hematology.nl. Accessed 19 April 20.

33. International Myeloma Society. International Myeloma Society Recommendations for the Management of Myeloma Patients During the COVID-19 Pandemic. https://cms.cws.net/content/ beta.myelomasociety.org/files/IMS\%20recommendations\%20for \%20Physicians\%20Final.pdf Accessed 19 April 2020.

34. Cortiula F, Pettke A, Bartoletti M, Puglisi F, Helleday T. Managing COVID-19 in the oncology clinic and avoiding the distraction effect. Ann Oncol. 2020;31:553-555.

35. Ueda M, Martins R, Hendrie PC, McDonnell T, Crews JR, Wong TL, et al. Managing cancer care during the COVID-19 pandemic: agility and collaboration toward a common goal. J Natl Compr Canc Netw. 2020: 1-4. https://doi.org/10.6004/jnccn.2020.7560. [Epub ahead of print].

36. Willan J, King AJ, Hayes S, Collins GP, Peniket A. Care of haematology patients in a COVID-19 epidemic. Br J Haematol. 2020;189:241-3.

37. von Lilienfeld-Toal M, Vehreschild J, Cornely O, Pagano L, Compagno F, EHA Infectious Disease Scientific Working Group, et al. Frequently asked questions regarding SARS-CoV2 in cancer patients-recommendations for clinicians caring for patients with malignant diseases. Leukemia. 2020; in press. https://doi.org/10. 1038/s41375-020-0832-y. [Epub ahead of print].

38. Mian H, Grant SJ, Engelhardt M, Pawlyn C, Bringhen S, Zweegman S, et al. Caring for older adults with multiple myeloma during the COVID-19 Pandemic: Perspective from the International Forum for Optimizing Care of Older Adults with Myeloma. J Geriatri Oncol. 2020. pii: S1879-4068(20)30190-9. https://doi. org/10.1016/j.jgo.2020.04.008. [Epub ahead of print].

39. Barr H, Dempsey J, Waller A, Huang Y, Williams N, Sharma N, et al. Ninety-minute daratumumab infusion is safe in multiple myeloma. Leukemia. 2018;32:2495-518.

40. Terpos E, Morgan G, Dimopoulos MA, Drake MT, Lentzsch S, Raje $\mathrm{N}$, et al. International Myeloma Working Group recommendations for the treatment of multiple myeloma-related bone disease. J Clin Oncol. 2013;31:2347-57.

41. Kyriakou C, Molloy S, Vrionis F, Alberico R, Bastian L, Zonder JA, et al. The role of cement augmentation with percutaneous vertebroplasty and balloon kyphoplasty for the treatment of vertebral compression fractures in multiple myeloma: a consensus statement from the International Myeloma Working Group (IMWG). Blood Cancer J. 2019;9:27.

42. Rajkumar SV, Dimopoulos MA, Palumbo A, Blade J, Merlini G, Mateos MV, et al. International Myeloma Working Group 
updated criteria for the diagnosis of multiple myeloma. Lancet Oncol. 2014;15:e538-548.

43. Ntanasis-Stathopoulos I, Gavriatopoulou M, Kastritis E, Terpos E, Dimopoulos MA. Multiple myeloma: Role of autologous transplantation. Cancer Treat Rev. 2020;82:101929.

44. Moreau P, Attal M, Hulin C, Arnulf B, Belhadj K, Benboubker L, et al. Bortezomib, thalidomide, and dexamethasone with or without daratumumab before and after autologous stem-cell transplantation for newly diagnosed multiple myeloma (CASSIOPEIA): a randomised, open-label, phase 3 study. Lancet. 2019;394:29-38.

45. Yimer H, Melear J, Faber E, Bensinger WI, Burke JM, Narang M, et al. Daratumumab, bortezomib, cyclophosphamide and dexamethasone in newly diagnosed and relapsed multiple myeloma: LYRA study. Br J Haematol. 2019;185:492-502.

46. Rajkumar SV. Multiple myeloma: 2020 update on diagnosis, riskstratification and management. Am J Hematol. 2020;95:548-67.

47. Rosinol L, Oriol A, Rios R, Sureda A, Blanchard MJ, Hernandez MT, et al. Bortezomib, lenalidomide, and dexamethasone as induction therapy prior to autologous transplant in multiple myeloma. Blood. 2019;134:1337-45.

48. Attal M, Lauwers-Cances V, Hulin C, Leleu X, Caillot D, Escoffre M, et al. Lenalidomide, bortezomib, and dexamethasone with transplantation for myeloma. $\mathrm{N}$ Engl $\mathrm{J}$ Med. 2017;376:1311-20.

49. Dimopoulos MA, Leleu X, Palumbo A, Moreau P, Delforge M, Cavo M, et al. Expert panel consensus statement on the optimal use of pomalidomide in relapsed and refractory multiple myeloma. Leukemia. 2014;28:1573-85.

50. Attal M, Richardson PG, Rajkumar SV, San-Miguel J, Beksac M, Spicka I, et al. Isatuximab plus pomalidomide and low-dose dexamethasone versus pomalidomide and low-dose dexamethasone in patients with relapsed and refractory multiple myeloma (ICARIA-MM): a randomised, multicentre, open-label, phase 3 study. Lancet. 2019;394:2096-107.

51. Dimopoulos MA, Dytfeld D, Grosicki S, Moreau P, Takezako $\mathrm{N}$, Hori $\mathrm{M}$, et al. Elotuzumab plus pomalidomide and dexamethasone for multiple myeloma. $N$ Engl $\mathrm{J}$ Med. 2018:379:1811-22.

52. Chari A, Vogl DT, Gavriatopoulou M, Nooka AK, Yee AJ, Huff $\mathrm{CA}$, et al. Oral selinexor-dexamethasone for triple-class refractory multiple myeloma. N Engl J Med. 2019;381:727-38.

53. Lonial S, Lee HC, Badros A, Trudel S, Nooka AK, Chari A, et al. Belantamab mafodotin for relapsed or refractory multiple myeloma (DREAMM-2): a two-arm, randomised, open-label, phase 2 study. Lancet Oncol. 2020;21:207-21.
54. Mateos MV, Hernandez MT, Giraldo P, de la Rubia J, de Arriba F, Lopez Corral L, et al. Lenalidomide plus dexamethasone for high-risk smoldering multiple myeloma. $\mathrm{N}$ Engl $\mathrm{J}$ Med. 2013;369:438-47.

55. Lonial S, Jacobus S, Fonseca R, Weiss M, Kumar S, Orlowski $\mathrm{RZ}$, et al. Randomized trial of lenalidomide versus observation in smoldering multiple myeloma. J Clin Oncol. 2020;38:1126-37.

56. Lakshman A, Rajkumar SV, Buadi FK, Binder M, Gertz MA, Lacy MQ, et al. Risk stratification of smoldering multiple myeloma incorporating revised IMWG diagnostic criteria. Blood Cancer J. 2018;8:59.

57. Fotiou D, Gavriatopoulou M, Terpos E. Multiple myeloma and thrombosis: prophylaxis and risk prediction tools. Cancers (Basel). 2020;12. pii: E191. https://www.ncbi.nlm.nih.gov/ pubmed/?term $=31940972$.

58. Larocca A, Cavallo F, Bringhen S, Di Raimondo F, Falanga A, Evangelista A, et al. Aspirin or enoxaparin thromboprophylaxis for patients with newly diagnosed multiple myeloma treated with lenalidomide. Blood. 2012;119:933-9. quiz 1093

59. Raje N, Vescio R, Montgomery CW, Badros A, Munshi N, Orlowski R, et al. Bone marker-directed dosing of zoledronic acid for the prevention of skeletal complications in patients with multiple myeloma: results of the Z-MARK study. Clin Cancer Res. 2016;22:1378-84.

60. Terpos E, Ntanasis-Stathopoulos I, Dimopoulos MA. Myeloma bone disease: from biology findings to treatment approaches. Blood. 2019;133:1534-9.

61. de Paula BHR, Araujo I, Bandeira L, Barreto N, Doherty GJ. Recommendations from national regulatory agencies for ongoing cancer trials during the COVID-19 pandemic. Lancet Oncol. 2020;21:624-7.

62. Mateos MV, Nahi H, Legiec W, Grosicki S, Vorobyev V, Spicka I, et al. Subcutaneous versus intravenous daratumumab in patients with relapsed or refractory multiple myeloma (COLUMBA): a multicentre, open-label, non-inferiority, randomised, phase 3 trial. Lancet Haematol. 2020;7:e370-80.

63. Elens L, Langman LJ, Hesselink DA, Bergan S, Moes D, Molinaro $\mathrm{M}$, et al. Pharmacologic treatment of transplant recipients infected with SARS-CoV-2: considerations regarding therapeutic drug monitoring and drug-drug interactions. Ther Drug Monit. 2020. https:// doi.org/10.1097/FTD.0000000000000761. [Epub ahead of print].

64. Roden DM, Harrington RA, Poppas A, Russo AM. Considerations for drug interactions on QTc in exploratory COVID-19 (Coronavirus Disease 2019) treatment. J Am Coll Cardiol. 2020. pii: S0735-1097(20)34918-4. https://doi.org/10.1016/j.jacc.2020. 04.016. [Epub ahead of print].

\section{Affiliations}

\section{Evangelos Terpos $\mathbb{D}^{1} \cdot$ Monika Engelhardt ${ }^{2} \cdot{\text { Gordon } \text { Cook }^{3} \cdot \text { Francesca Gay }}^{4}$ - Maria-Victoria Mateos $\mathbb{1}^{5,6}$. loannis Ntanasis-Stathopoulos $\mathbb{1}^{1} \cdot$ Niels W. C. J. van de Donk ${ }^{7} \cdot$ Hervé Avet-Loiseau $^{8} \cdot$ Roman Hajek $^{9}$. Annette Juul Vangsted (10 ${ }^{10} \cdot$ Heinz Ludwig $\mathbb{1}^{11} \cdot$ Sonja Zweegman ${ }^{7} \cdot$ Philippe Moreau $^{12} \cdot$ Hermann Einsele $^{13}$. Mario Boccadoro $^{4} \cdot$ Jesus San Miguel $^{14} \cdot$ Meletios A. Dimopoulos ${ }^{1} \cdot$ Pieter Sonneveld $^{15}$}

1 Department of Clinical Therapeutics, School of Medicine, National and Kapodistrian University of Athens, Athens, Greece

2 Faculty of Freiburg, Hematology and Oncology Department, Interdisciplinary Cancer Center (ITZ) and Comprehensive Cancer Center Freiburg (CCCF), University of Freiburg,

Freiburg, Germany
3 Leeds Cancer Centre, Leeds Teaching Hospitals National Health Service Trust and University of Leeds, Leeds, UK

4 Division of Hematology, University of Turin, Azienda Ospedaliero-Universitaria Città della Salute e della Scienza di Torino, Turin, Italy 
5 Cancer Research Unit, University Hospital of Salamanca, Instituto de Investigación Biomédica de Salamanca (IBSAL), Salamanca, Spain

6 Institute of Cancer Molecular and Cellular Biology (USAL-CSIC), Centre for Cancer Research (IBMCC), Instituto de Investigación Biomédica de Salamanca (IBSAL), Salamanca, Spain

7 Department of Hematology, Cancer Center Amsterdam, Amsterdam UMC, Vrije Universiteit Amsterdam, Amsterdam, The Netherlands

8 Genomics of Myeloma Laboratory, L'Institut Universitaire du Cancer Oncopole, Toulouse, France

9 Department of Hemato-Oncology, University Hospital Ostrava and Faculty of Medicine, University of Ostrava, Ostrava, Czech Republic
10 Department of Hematology, Copenhagen University Hospital, Rigshospitalet, Copenhagen, Denmark

11 Wilhelminen Cancer Research Institute, c/o Department of Medical Oncology, Hematology and Palliative Care, Wilhelminenspital Wien, Austria

12 Department of Hematology, University Hospital Hotel-Dieu, Nantes, France

13 Department of Internal Medicine II, University Hospital of Würzburg, Würzburg, Germany

14 Clínica Universidad de Navarra-Centro de Investigación Médica Aplicada, Instituto de Investigación Sanitaria de Navarra, Centro de Investigación Biomédica en Red de Cáncer, Pamplona, Spain

15 Department of Hematology, Erasmus MC Cancer Institute, Rotterdam, The Netherlands 